\title{
POLICY USAGE IN GMPLS OPTICAL NETWORKS
}

\author{
Belkacem Daheb ${ }^{1,2}$, Guy Pujolle ${ }^{1}$ \\ ' University of Paris 6, LIP6 Lab, 8, rue du Capitaine Scott, 75015 Paris ; ${ }^{2}$ Institut Supérieur \\ d'Electronique de Paris (ISEP) ; \{Belkacem.Daheb,Guy.Pujolle\}@lip6.fr
}

\begin{abstract}
Recently, a great consortium on Generalized Multi Protocol Label Switching (GMPLS) is emerging as the control plane for next generation optical backbone networks. This article proposes guidelines for managing optical networks controlled by GMPLS in a policy based fashion. A flexible management solution is presented especially for optical network issues, where the service management system efficiently impacts the control plane offering the possibility to dynamically change network functionality to enhance the controllability of optical networks.
\end{abstract}

Key words: GMPLS, Optical Networks, Policy based Management, Service Management.

\section{INTRODUCTION}

In recent years, there has been a dramatic increase in data traffic, driven primarily by the explosive growth of the Internet as well as the proliferation of virtual private networks (VPN). On the other hand the huge amount of bandwidth, offered by optical links, render optical networks the ideal candidate for next generation backbone networks.

The initial use for optical fiber communication and its prevalent use today, is to provide high-bandwidth point-to-point pipes. At the ends of these pipes, data is converted from the optical to the electrical domain, and all the switching, routing, and intelligent control functions are handled by higherlayer equipment, such as SONET or IP boxes ${ }^{1}$.

With IP routers emerging as among the dominant clients of the optical layer, there has been a great deal of interest recently in trying to obtain a closer interaction between the IP layer and the optical layer from a control and management perspective. This is done using distributed control 
protocols, which are widely used in data communications networks such as IP and ATM. These protocols set up and take down connections, maintain topology databases, and perform route computations. In any event, there is emerging consensus on basing these optical layer protocols on a modified version of Multi-Protocol Label Switching (MPLS), now called Generalized MPLS $^{2}$ (GMPLS). One of the key aspects of MPLS is the addition of a new connectivity abstraction: explicitly routed point-to-point path. This is accomplished by the concept of explicitly routed label switched paths (LSP).

Nonetheless, maturing distributed optical intelligence solutions lead to a new control-management interaction scheme. In addition, since the control plane affects pivotal functions in classical network management, it is instrumented to fit only a question-answer relation with that centralized management plane ${ }^{3}$. To accomplish an efficient control-management interoperation, i.e. to reduce management complexity and delay and to enhance automation, one of the most commonly used approaches is the policy-based management (PBM). An effort of standardization has been made on the field of PBM at the IETF $^{4,5}$. The PBM approach provides an overall, networkwide regulatory infrastructure and allows network administrators and Service Providers to simplify end-to-end configuration and regulation of the network behavior with enhanced Quality of Service (QoS) and Traffic Engineering (TE) features rather than configure individual devices. For instance, network administrators may find a way to preprogram their networks through rules or policies that define how the network should automatically respond to various networking situations.

To wrap up things, Figure 1 shows a layered architecture of optical networks, independently of the transmission technology. In the middle stems a Data Plane, which forms the data transmission mechanisms based on wavelength paths. This plane is over hanged by a Control Plane which in turn processes generic provisioning mechanisms, that are independent from transmission ones. On the bottom of the figure, we have a management plane which performs management tasks that are less real-time than the previous.

This paper provides a brainstorming on the integration of the policy management approach to immerging optical networks controlled by a GMPLS control plane. Next section addresses the possible policy based interaction between the control and management plane. It is followed by a Section that details the different mechanisms involved in controlling such optical networks. While the last section details every component of the control plane by showing where would policies impact. 


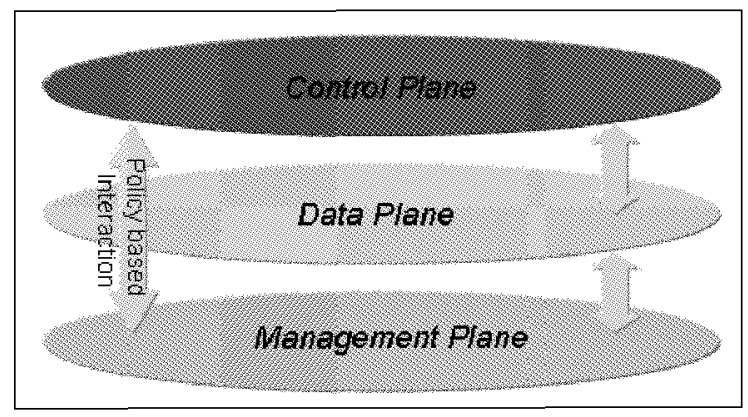

Figure 1. Optical network functional planes

\section{CONTROL AND MANAGEMENT PLANE INTERACTION}

Emerging requirements brought by the growing amount data traffic and need of automation lead to the introduction of GMPLS as a control plane for automating the provisioning process. GMPLS allows the control of optical Label Switched Paths (LSP) closer to the data plane than the management plane. Because GMPLS is an extension from MPLS to non packet switched networks, and further difficulties come from the optical layer. For example, optical network may contain entirely photonic, hybrid, and opaque network nodes.

Consequently, basic control plane functionality - carrying out autonomous topology management, routing, signaling, and required QoS enforcement for traffic engineering - raises some issues of consistency when applied without appropriate inter-operations with the management plane, and constitute a challenge to efficient network management. On the other hand, in regard to configuration management and especially in provisioning, the manual establishment of explicit LSPs with associated QoS parameters is slow, error prone, and laborious to network administrators.

Previous studies tend to the introduction of a policy-based management system on MPLS networks, ${ }^{6,7,9}$. This proves that even though MPLS efficiently controls a network but higher abstraction layer mechanisms are needed for the controllability of LSP life cycle. Since GMPLS brings the opportunity to establish network wide paths within the optical network, and therefore implicitly offer a rationale and also a background for end to end service deployment, a reinforced management system including a Policybased Management (PBM) infrastructure is adjoined to the previous functional planes structuring, see Figure 1. Globally PBM is viewed as part 
of the management plane. It deals specifically with node intelligence configuration, decision at network level, and with service related actions. A policy controlled based management system can operate at a higher, more service focused level where control for admission and security as well as QoS are orchestrated in accordance with parameters negotiated in different service level agreements ${ }^{10}$.

On the other hand, even though it seems that network management forms a homogeneous unit, there is a clear separation between the traditional management functionality and dynamic management. The traditional management of GMPLS networks is to manage GMPLS network elements the TMN way, achieving the so called, Fault, Configuration, Accounting, Performance, and Security (FCAPS) management processes. The dynamic management tasks are performed essentially via the policy-based management system. The former can be seen as unaware to the optical nature while the later is devoted, amongst other, to handle different constraints, especially optical ones.

\section{GMPLS CONTROL PLANE}

Generalized multi-protocol label switching, also referred to as GMPLS, supports not only devices that perform packet switching, but also those that perform switching in the time, wavelength, and space domains. This part briefly presents the traffic engineering (TE) features introduced by GMPLS to extend the MPLS control plane to non packet networks.

The GMPLS protocol suite could be devised into two blocks: a routing block for choosing the route that must take the traffic and a signaling one that allocate resources for the path chosen in the routing step, Figure 2. Since GMPLS has two building blocks, the TE enhancements affect both of them $^{11,12}$.

Some of these enhancements concern the routing block ${ }^{11}$, and are cited in what follows. Among the routing enhancements, the LSP hierarchy is the notion that LSP of different types (FSC, LSC, TDMC, PSC) can be nested inside others. At the top of this hierarchy are nodes that have fiber-switchcapable (FSC) interfaces, followed by nodes that have lambda-switchcapable (LSC) interfaces, followed by nodes that have TDM-capable interfaces, followed by nodes with packet-switch-capable (PSC) interfaces. One of the most important enhancements is Link Bundling, which means aggregating several links of similar characteristics, and assigning these aggregated attributes to a single "bundled" link. In so doing, the size of the link state database (maintained by the routing protocol, OSPF-TE for instance) is reduced by a large factor. Especially in optical networks, with a 
large number of links and ports on each router, an Unumbered Link feature allows to locally number some links and ports to prevent the lake of addresses.

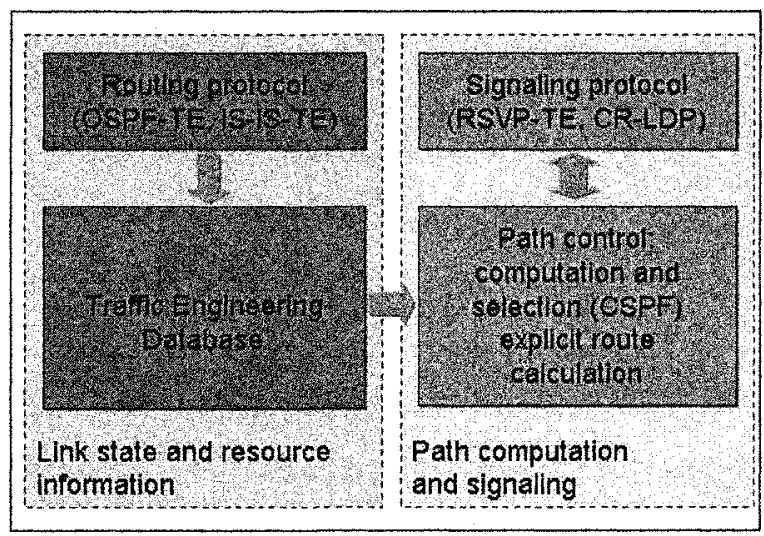

Figure 2. GMPLS building blocks

While in the signaling part a new feature named the Hierarchical LSP Setup, has been introduced. It means that an LSP could encompass several regions and take advantage of the link bundling respecting the LSP Hierarchy defined. In optical networks, the signaling protocol is responsible of choosing the adequate wavelength on each link maintaining as much as possible the wavelength continuity constraint. The set of wavelengths chosen define the different labels of an optical LSP. GMPLS signaling allows a label to be suggested by an upstream node, thanks to the Suggested Label. In the basic MPLS architecture, LSP are unidirectional; but Bidirectional optical LSP (or lightpaths) are a requirement for many optical networking service providers. This is possible in GMPLS thanks to the Bidirectional LSP setup. The last enhancement to signaling is the Notify Messages that are sent to notify the node(s) responsible for restoring the connections when failures occur.

It is expected that in very large networks, such that controlled by a GMPLS control plane, a network operator could face many failures. To facilitate the management of the occurred failures a Link Management Protocol (LMP) was added to the GMPLS protocol suit. A key service provided by LMP is to set-up and verify associations between neighboring nodes.

One of the merits of GMPLS stems from its ability to automate circuit provisioning in optical networks. Connection management complexity related to LSP setup/modification/teardown is thus reduced. This simplification is realized through a suite of protocol extensions currently under standardization in the $\mathrm{IETF}^{2}$. 
Figure 2 presents the functional GMPLS building blocks that would be distributed along the different network nodes. The link state Internet Gateway Protocol (IGP), which can be either OSPF or Intermediate System to Intermediate System (IS-IS) with optical-specific extensions, is responsible for distributing information about optical topology, resource availability, and network status. This information is then stored in a traffic engineering (TE) database. A constraint-based routing function acting as a path selector is used to compute routes for the desired LSP. This route calculation accounts for the information collected in the TE database as well as the traffic requirements. Once the route has been computed, a signaling protocol such as Resource Reservation Protocol with TE (RSVP-TE) is used for path activation (i.e., instantiation of the label forwarding state along the computed path).

For instance, as stated before, the GMPLS control plane is capable of performing fault and connection management in a fast distributed way. The service provider will thus be able to quickly and efficiently build highcapacity optical infrastructures supporting fast connection provisioning. Hence, new types of services requiring stringent connection setup times such as bandwidth-on-demand services would have the desired fast deployment time.

\section{USING POLICIES WITH GMPLS}

The GMPLS technology enables the setup of Label Switched Paths (LSP) through an optical network. Initially, the idea of GMPLS was to automate the service provisioning and apply traffic engineering. However, in the meantime traffic engineering and QoS in optical networks became the dominant driving force behind GMPLS. But GMPLS can't deal with all aspects of QoS or TE expected, since it is not guided by a higher level abstraction system to assist it reaching the goal of gracefully managing and controlling a network. To apply policy-based management concepts to manage a GMPLS network is an appropriate way of dealing with large sets of managed elements instead of manually managing each network element ${ }^{3}$.

With regard to the previous section it is clear that the GMPLS control plane must be guided with some directives expressed under the form of policy rules. But what would policies impact in the GMPLS control plane?

To answer this question, let's go back to Figure 2 that represents the building blocks of an optical node controller. Policies would mainly impact these boxes. Requirements and conditions of policy usage are exemplified for each block in what follows through provisioning cases with respect to optical networks. 


\subsection{Routing Policies}

This set of policies translates various types of constraints provided by the network operator and the client expectations such as constraints related to performance and business model.

The client expectations would introduce constraints on the path selection. A client may require a certain boundary to the time taken to establish his connection. He may also require a specified protection for his connection or even a restoration instead. Knowing that the operator applies rerouting of some connections from time to time, in order to enhance the performance of his network, a client can forbid to the operator rerouting his connection. Or at least he can limit the number of rerouting of his connection for a period of time. A client may also ask for certain confidentiality, in order to be sure that his traffic cannot be read by an intruder. Finally, the client can ask for a quality connection respecting some quality of signal features of the wavelength (BER for example). These constraints can be inferred through Service Level agreements ${ }^{10}$.

It is clear that these user constraints participate in the choice of the path taken by a LSP. To handle these constraints lets go more in detail with a look at the GMPLS path computation process. The GMPLS path computation is performed by a Constraint Shortest Path First (CSPF) algorithm which uses the link state database maintained by the routing algorithm and some other constraints applied to the Shortest Path First algorithm. These constraints are directly derived from the user constraints described above and some other constraints defined by the administrator. The path computation is based on a Constraint Based Routing which computes an explicit route on the originating node and passes this information to the signaling protocol which is responsible of setting up the light-path.

\subsection{Signaling Policies}

After choosing the path for a connection, a signaling phase consists in choosing along the path the adequate wavelength used on each link. The main goal of this step is to reduce the wavelength conversion and minimize the set of wavelength needed in an optical network.

There are different schemes to perform wavelength selection named Spectral Routing in Papadimitriou ${ }^{13}$. The spectral routing is subject to constraints that may be a combination of both external and internal (or intrinsic) constraints due to the physical transmission medium for instance. Notice that external spectral routing constraints are generally dictated by Bit Error Rate (BER) or inferred through Service Levels. As a matter of fact, 
external constraints must be injected into the node performing the spectral routing; this is the role of the signaling policies.

More over, a spectral route can be computed by the ingress node, or each hop of the path or even by the egress node, depending on the scheme used. In each scheme, a node must choose and propose a set of possible wavelengths to use. This set is computed based on the set of available wavelengths and the set of wavelengths proposed by the ingress node. However, the choice is not trivial and can be guided via policies. For instance, the network operator can forbid the use of a wavelength and reserve it for signaling purposes.

\subsection{Dimensioning policies}

For optimization reasons, it may be desirable for a photonic crossconnect to optically switch multiple wavelengths as a unit. This unit is a waveband that represents a set of contiguous wavelengths, which can be switched together to a new waveband ${ }^{2}$. Hence, another stage is added in the LSP hierarchy, defined above. In Noirie ${ }^{14}$, it is shown that the waveband stage reduces the complexity and cost of the optical cross-connects. Waveband coverage of the network is given first before routing the wavelength LSP. Every waveband setup is declared as a Forwarding Adjacency by the routing protocol. By definition, a Forwarding Adjacency (FA) is a TE link between two GMPLS nodes whose path transits one or more other (G)MPLS nodes in the same instance of the (G)MPLS control plane $^{2}$. In other words, the wavebands are seen as links by the nodes; hence, they constitute the virtual topology over which the wavelength LSP will be setup.

This way of constructing the topology is static. The waveband coverage is based on some statistical traffic analysis but cannot predict and fit all the future connections demand. Therefore, a more dynamic way of constructing the topology is needed. For instance, it would be interesting to add some wavebands during the network life cycle.

Dimensioning policies are a mean to manage the virtual topology. Their role is to setup, modify or even tear down some wavebands depending on the network state and the connections demand. For example, when the whole wavebands are over loaded, a policy is needed to add a new waveband in order to permit future wavelength LSP establishment.

Another issue concerns the bundling of existing wavelength into wavebands during the waveband coverage step, which is not trivial. Two complementary nesting strategies have long been studied in Noirie ${ }^{14}$. The first grooming strategy consists in nesting lambda-LSP in a single waveband-LSP from end to end. The second grooming strategy consists in 
nesting a common sub-path of wavelength LSP into a waveband. However, the choice of the bundling strategy is not made at the node level but at higher levels. The dimensioning policies can be used to guide the grooming strategy from a higher level.

Finally, in order to enhance the flexibility of network configuration, the waveband construction must be policy-guided.

\subsection{Logical Policy Levels}

Having presented some directives that can be applicable for a policy management of GMPLS optical networks, here a logical view of these policies is inferred, Figure 3. The lowest level is concerned with the optical device-level configuration, the mid-level deals with the network-wide (GMPLS) configurations, and the upper layer handles services.

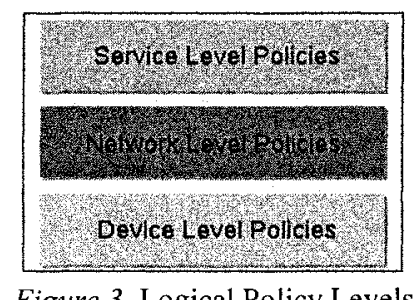

Figure 3. Logical Policy Levels

The device level policies deal essentially with optical network element configuration. It consist mainly in configuring the switching matrix of the optical cross-connects. The upper level policies are defined more network wide and include routing, signaling and dimensioning policies. These kinds of policies could eventually affect the GMPLS protocol stack, configuring both routing and signaling protocols the way described before. So, the control plane policies discussed above are a subset of these policies. The upper level defines policy rules depending on service exigencies that can emanate from service level agreements.

Notre that above the service oriented policies, we could have a higher level comprising some policies defined by the administrator itself, named Business Level Policies. These policies would respond to the manner that the administrator want to use his network and could be totally independent from service or network objectives.

\section{CONCLUSION}

This article dealt with policy based management of GMPLS optical networks. The need for policy rules was highlighted in optical networks 
controlled by GMPLS. The paper showed where and for what purpose do policies impact the GMPLS tool box. The policies discussed enhance the flexibility of provisioning and dimensioning of an optical network. We expect that this work will contribute in defining a policy based system for GMPLS optical networks.

\section{REFERENCES}

1. R. Ramaswami, Optical Fiber Communication: From Transmission To Networking, Invited Article, IEEE Communications Magazine, May 2002.

2. Eric Mannie, Generalized Multi-Protocol Label Switching Architecture, IETF draft, draftietf-ccamp-gmpls-architecture-07.txt, May 2003.

3. B. Berde, E. Dotaro, M. Vigoureux, Evolution of Policy-enabled GMPLS Optical Networking, Alcatel-CIT, Research and Innovation, Technical Report.

4. R. Yavatkar, D. Pendarakis, R. Guerin, A Framework for Policy-based Admission Control, Request for Comments: 2753, January 2000.

5. A. Westerinen, J. Schnizlein, J. Strassner, M. Scherling, B. Quinn, S. Herzog, A. Huynh, M. Carlson, J. Perry, S. Waldbusser, Terminology for Policy-Based Management, Request for Comments: 3198, November 2001.

6. S. Wright, S. Herzog, F. Reichmeyer, R. Jaeger, Requirements for Policy Enabled MPLS, IETF draft, draft-wright-policy-mpls-00.txt, March 2000.

7. M. Brunner, J. Quittek, MPLS Management using Policies, IEEE/IFIP, 2001.

8. K. Isoyama, M. Brunner, M. Yoshida, J. Quittek, R. Chadha, G. Mykoniatis, A. Poylisher, R. Vaidyanathan, A. Kind, F. Reichmeyer, Policy Framework MPLS Information Model for QoS and TE, IETF draft, draft-chadha-policy-mpls-te-01.txt, December 2000.

9. T. Hamada, P. Czezowski, T. Chujo, A Policy-enabled GMPLS-based Control Plane for Bandwidth Brokering, NOMS 2002 (IEEE2002).

10.B. Daheb, F. Wissam, M. Du-Pond, A. Olivier, B. Berde, M. Vigoureux, G. Pujolle, Service Level Agreement in Optical Networks, IEEE-IFIP Net-Con 2003, Kluwer ed.

11.A. Banerjee, J. Drake, J. Lang, B. Turner, K. Kompella, Y. Rekhter, Generalized Multiprotocol Label Switching: An Overview of Routing and Management Enhancements, IEEE Communications Magazine, January 2001.

12.A. Banerjee, J. Drake, J. Lang, B. Turner, D. Awduche, L. Berger, K. Kompella, Y. Rekhter, Generalized Multiprotocol Label Switching: An Overview of Signaling Enhancements and Recovery Techniques, IEEE Communications Magazine, July 2001.

13.D. Papadimitriou, M. Vigoureux, E. Dotaro, K. Shiomoto, E. Oki, N. Matsuura, Generalized MPLS Architecture for Multi-Region Networks, IETF draft, draft-vigoureuxshiomoto-ccamp-gmpls-mrn-00.txt, October 2002.

14.L. Noirie, M. Vigoureux, E. Dotaro, Impact of Intermediate Traffic Grouping on the Dimensioning of Multi-Granularity Optical Networks, Proc. OFC 2001. 